\title{
Metadherin facilitates podocyte apoptosis in diabetic nephropathy
}

\author{
Wen-Ting Liu, ${ }^{1,4}$, Fen-Fen Peng ${ }^{1,4}$, Hong-Yu Li ${ }^{1,4}$, Xiao-Wen Chen ${ }^{2}$, Wang-Qiu Gong', Wen-Jing Chen ${ }^{1}$, Yi-Hua Chen ${ }^{1}$, Pei-Lin Li ${ }^{1}$, \\ Shu-Ting Li ${ }^{1}$, Zhao-Zhong $\mathrm{Xu}^{3}$ and Hai-Bo Long ${ }^{*, 1}$
}

Apoptosis, one of the major causes of podocyte loss, has been reported to have a vital role in diabetic nephropathy (DN) pathogenesis, and understanding the mechanisms underlying the regulation of podocyte apoptosis is crucial. Metadherin (MTDH) is an important oncogene, which is overexpressed in most cancers and responsible for apoptosis, metastasis, and poor patient survival. Here we show that the expression levels of Mtdh and phosphorylated p38 mitogen-activated protein kinase (MAPK) are significantly increased, whereas those of the microRNA-30 family members (miR-30s) are considerably reduced in the glomeruli of DN rat model and in high glucose (HG)-induced conditionally immortalized mouse podocytes (MPC5). These levels are positively correlated with podocyte apoptosis rate. The inhibition of Mtdh expression, using small interfering RNA, but not Mtdh overexpression, was shown to inhibit HG-induced MPC5 apoptosis and p38 MAPK pathway, and Bax and cleaved caspase 3 expression. This was shown to be similar to the effects of p38 MAPK inhibitor (SB203580). Furthermore, luciferase assay results demonstrated that Mtdh represents the target of miR-30s. Transient transfection experiments, using miR-30 microRNA (miRNA) inhibitors, led to the increase in Mtdh expression and induced the apoptosis of MPC5, whereas the treatment with miR-30 miRNA mimics led to the reduction in Mtdh expression and apoptosis of HG-induced MPC5 cells in comparison with their respective controls. Our results demonstrate that Mtdh is a potent modulator of podocyte apoptosis, and that it represents the target of miR-30 miRNAs, facilitating podocyte apoptosis through the activation of HG-induced p38 MAPK-dependent pathway.

Cell Death and Disease (2016) 7, e2477; doi:10.1038/cddis.2016.335; published online 24 November 2016

Podocyte loss is emerging as a central pathological mechanism underlying diabetic nephropathy (DN). Podocyte apoptosis is one of the major causes of podocyte loss ${ }^{1}$ and one of the earliest cellular lesions affecting the diabetic kidney. ${ }^{2}$ Recent studies revealed that many pathways are involved in this process, including Notch, ${ }^{3}$ transforming growth factor $\beta$ (TGF- $\left.\beta\right),{ }^{4}$ and p38 mitogen-activated protein kinase (MAPK) ${ }^{2,5}$ signaling pathways. However, cellular and molecular pathomechanisms responsible for podocyte apoptosis in DN remain largely unknown.

Here we evaluated the involvement of metadherin (Mtdh, also known as astrocyte elevated gene-1 (AEG-1) ${ }^{6}$ or lysine-rich CEACAM1 co-isolated (LYRIC) ${ }^{7}$ ) in this process. A comprehensive and convincing body of data highlights MTDH as an important oncogene that is overexpressed in all previously investigated cancers. ${ }^{8}$ It is involved in cancer cell proliferation, survival, autophagy, migration, invasion, apoptosis, angiogenesis, metastasis, and treatment resistance. ${ }^{9}$ In addition to its role in tumorigenesis, $\mathrm{MTDH}$ is a pleiotropic protein involved in various physiological and pathological processes, including development, ${ }^{10}$ neurodegeneration, ${ }^{11}$ inflammation, ${ }^{12-14}$ and epithelial-mesenchymal transition (EMT). ${ }^{15} \mathrm{MTDH}$ has a role in these processes through the regulation of various signaling pathways, including transcription factor nuclear factor-kB (NF-kB), GTPase HRAS, phosphoinositide 3-kinase (PI3K)/AKT, p38 MAPK, and WNT pathways. ${ }^{15,16}$ Recently, Eisenrech et al. ${ }^{17}$ demonstrated that podoplanin, a factor involved in cancer biology but poorly characterized and functionally analyzed in podocytes, is antiapopotic in Angll-induced human podocytes, and regulated by miR-29b. However, the expression and role of MTDH in podocytes have not been investigated before.

p38 MAPK is one of the important member of MAPKs, activated by proinflammatory cytokines or cellular stress, such as genotoxic, osmotic, hypoxic, or oxidative stress. ${ }^{18}$ Previous studies have implicated p38 MAPK in a variety of cellular activities, including cell survival, death, proliferation, differentiation, and transformation. ${ }^{19}$ It was shown to regulate apoptosis by modulating the activities of the Bcl-2 family proteins. $^{20}$ Furthermore, P38 MAPK was implicated in podocyte apoptosis induced by doxorubicin (Adriamycin) ${ }^{21}$ and high serum lipopolysaccharide levels. ${ }^{22}$

Data obtained over the past decade have demonstrated that microRNAs (miRNAs) have vital roles in the cell growth, proliferation, differentiation, apoptosis, and stress response. ${ }^{23}$ Extensive investigations showed that $\mathrm{MTDH}$ represents a target gene of several miRNAs. ${ }^{16}$ miR-30 family consists of five members, miR-30a-miR-30e, and it was shown that they are involved in hepatocyte $\mathrm{EMT}^{24}$ cancer cell apoptosis and autophagy, ${ }^{25}$ and cardiomyocyte apoptosis. ${ }^{26}$ Moreover, $\mathrm{Li}$ et al. ${ }^{27}$ reported that the overexpression of miR-30a-5p inhibits cell proliferation, colony formation, and induces apoptosis in liver cancer cells by targeting MTDH. Members

\footnotetext{
${ }^{1}$ Department of Nephrology, ZhuJiang Hospital, Southern Medical University, Guangzhou 510280, China; ${ }^{2}$ Department of Nephrology, NanFang Hospital, Southern Medical University, Guangzhou 510515, China and ${ }^{3}$ Department of Emergency, ZhuJiang Hospital, Southern Medical University, Guangzhou 510280, China

*Corresponding author: H-B Long, Department of Nephrology, ZhuJiang Hospital, Southern Medical University, 253 Industrial Avenue, Guangzhou 510280, Guangdong, China. Tel: +86 206278 2305; Fax: +86 206278 2305; E-mail: longhb1966@163.com

${ }^{4}$ These authors contributed equally to this work.

Received 27.6.16; revised 23.9.16; accepted 23.9.16; Edited by A Stephanou
} 
of miR-30 family were shown to be abundantly expressed in podocytes, and the downregulation of their expression facilitates podocyte apoptosis in glomerulosclerosis. ${ }^{28}$

In this study, we investigated the potential involvement of Mtdh in podocyte apoptosis, together with the interactions between the members of miR-30 family and the changes in Mtdh expression. We demonstrate here that MAPK signaling is required for Mtdh-mediated induction of podocyte apoptosis.

\section{Results}

Apoptosis rate is increased DN and HG-induced MPC5 cells. The results obtained using electron microscopy showed the evidence of glomerular basement membrane thickening, effacement, and focal fusion of the podocyte foot process in $\mathrm{db} / \mathrm{db}$ mice (Figure 1a).

We evaluated podocyte apoptosis in $\mathrm{db} / \mathrm{db}$ and $\mathrm{db} / \mathrm{m}$ mice by terminal deoxynucleotidyl transferase dUTP nick end labeling (TUNEL) staining combined with WT-1 (podocyte nucleus marker) immunofluorescent staining. WT-1 immunostaining showed a significant reduction in the number of podocyte in the glomeruli of $\mathrm{db} / \mathrm{db}$ mice (Figures $1 \mathrm{~b}$ and $\mathrm{c}$ ). In contrast, a significant increase in the intensity of TUNEL staining of the podocytes in $\mathrm{db} / \mathrm{db}$ mice was observed in comparison with $\mathrm{db} / \mathrm{m}$ (Figures $1 \mathrm{~b}$ and $\mathrm{d}$ ). We next estimated the apoptosis rate of MPC5 cells treated with $5.3 \mathrm{mM}$ glucose
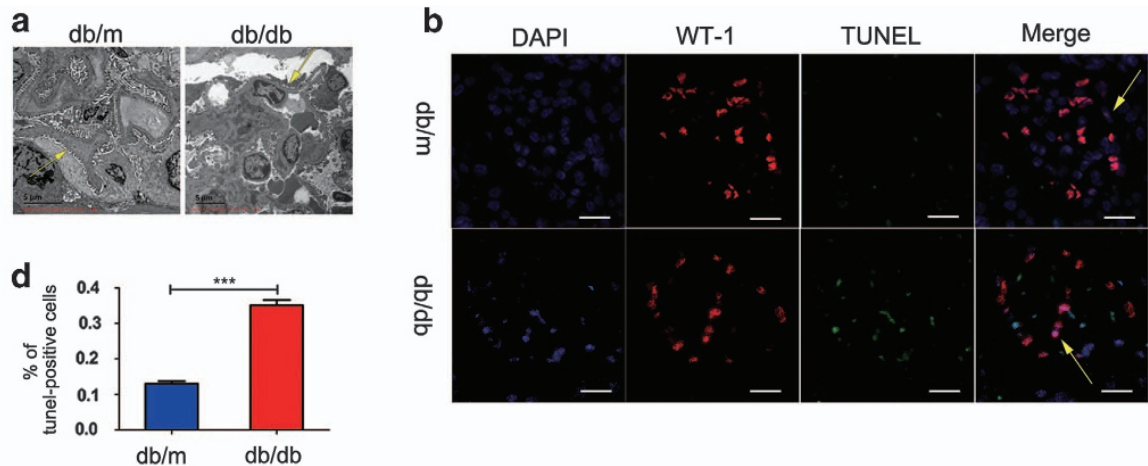

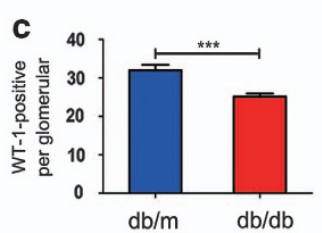

e
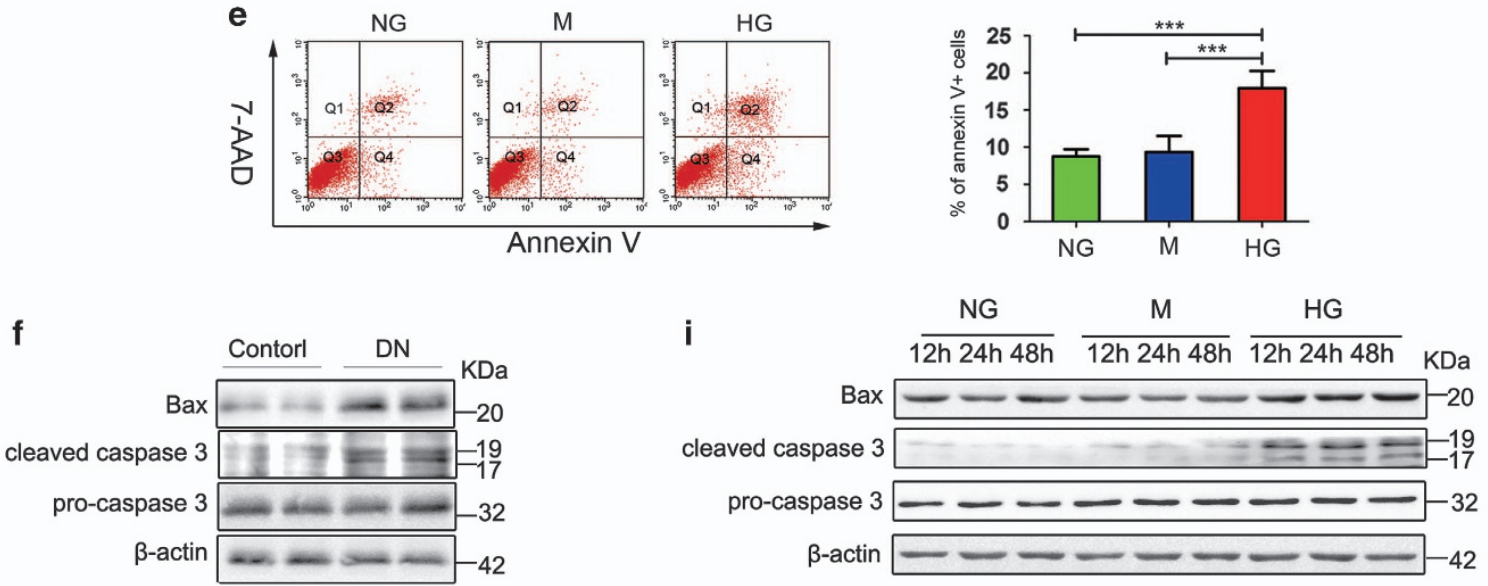

cleaved caspase 3

pro-caspase 3

$\beta$-actin

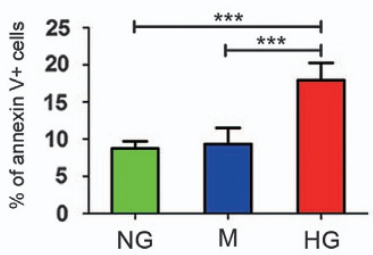

\section{$-$}
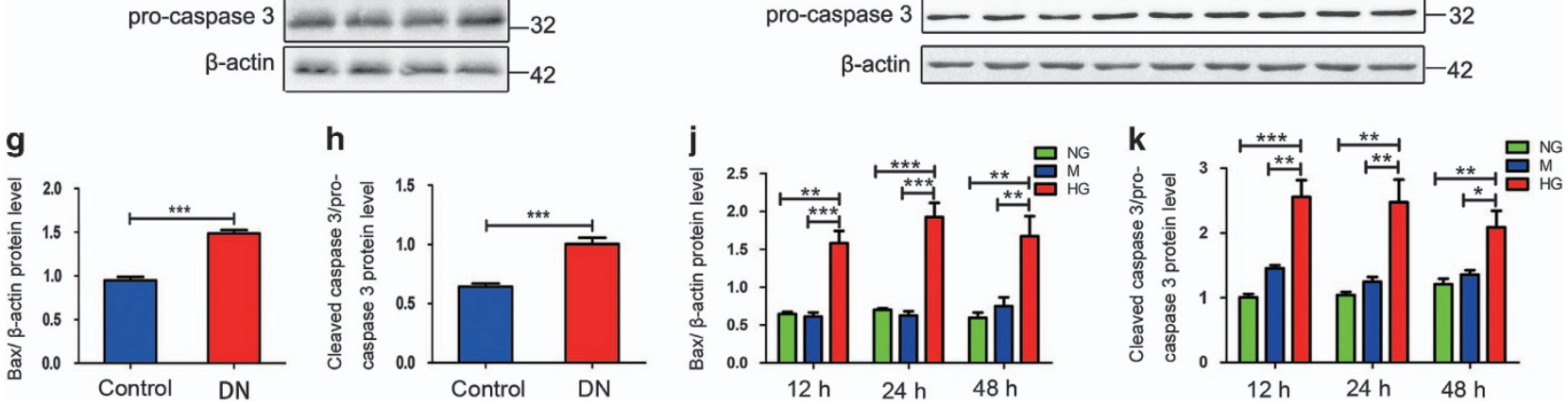

Figure 1 Apoptosis rate is increased DN and HG-induced MPC5 cells. (a) Thickening of glomerular basement membrane, and the shortening and effacement of podocyte foot process were observed in db/db mice (yellow arrows). Scale bars, $5 \mu \mathrm{m}$. (b) Representative images showing WT-1 and TUNEL double staining. Scale bars, $20 \mu \mathrm{m}$. (c) Quantification of WT-1-positive cells in glomerular sections. The number of WT-1-positive cells was obtained from three randomly selected glomeruli per kidney section ( $n=9$ per group). (d) Quantification of the podocyte apoptosis rate in kidney glomeruli of $\mathrm{db} / \mathrm{m}$ and $\mathrm{db} / \mathrm{db}$ mice. Values are presented as the percent of double-labeled/WT-1-labeled cells from three randomly selected glomeruli per kidney section $(n=9)$. (e) Apoptosis rate of MPC5 cells treated with NG, M, and HG for $48 \mathrm{~h}(n=4)$. (f) Immunoblotting for Bax and cleaved caspase 3 expression in glomeruli from streptozotocin-induced diabetic rats and the controls. (g-h) Quantification of Bax and cleaved caspase 3 expression in vivo $(n=6)$. (i) Bax and cleaved caspase 3 expression in MPC5 cells stimulated with NG, M, and HG for 12, 24, or $48 \mathrm{~h}$. (j-k) Quantification of the results presented in (I) ( $n=3$ ). Bars represent mean \pm S.E.M. ${ }^{*} P<0.05,{ }^{* *} P<0.01$, and ${ }^{\star * *} P<0.001$ 

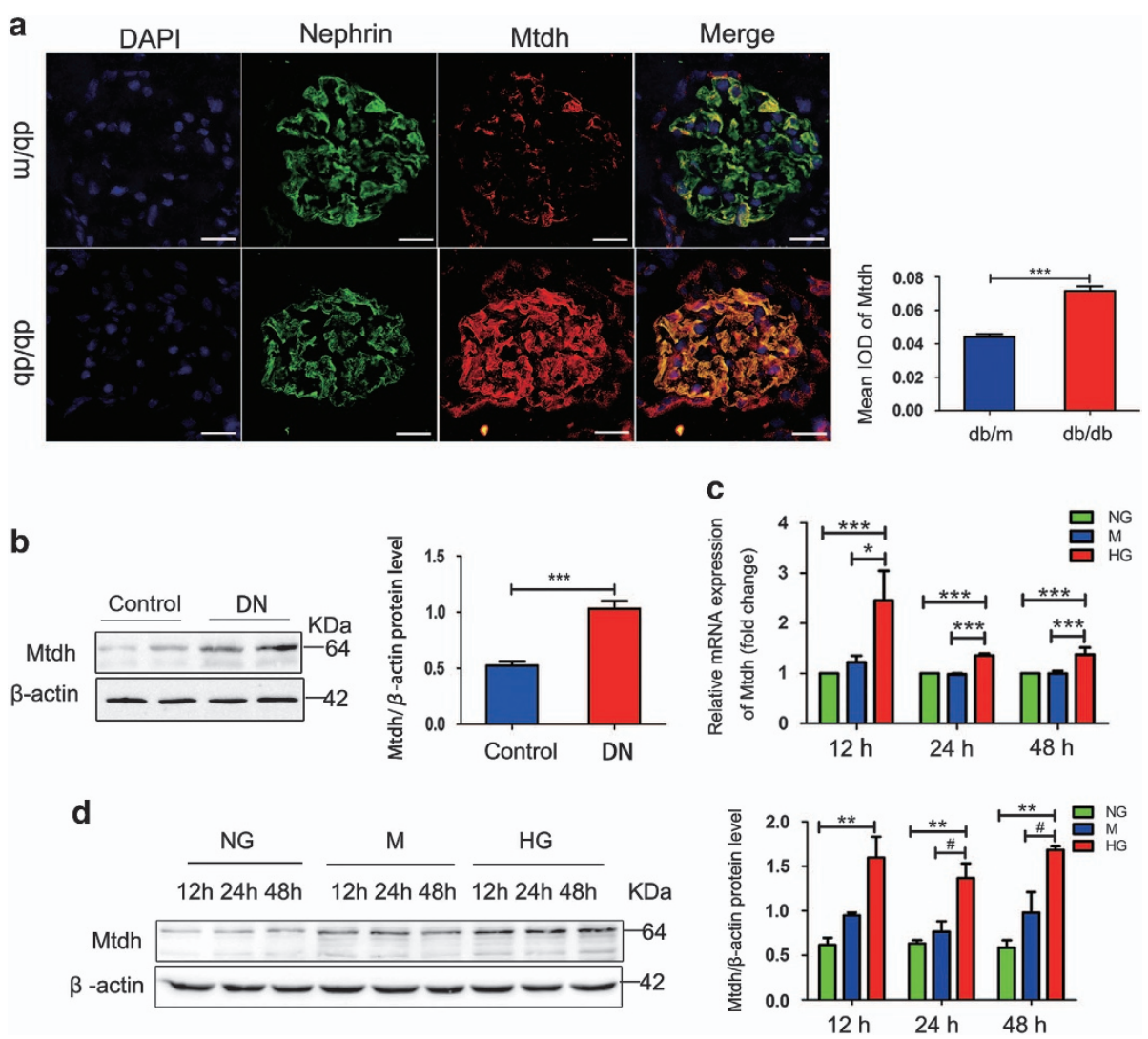

Figure 2 Mtdh expression is increased in vivo and in vitro models of DN. (a) Nephrin and Mtdh double staining. Scale bars, $20 \mu \mathrm{m}$. (b) Mtdh protein expression in the glomeruli of DN rats $(n=6)$. (c) Mtdh expression levels in MPC5 cells treated with NG, M, or HG for $48 \mathrm{~h}(n=3)$. (d) Mtdh protein expression levels in MPC5 cells treated with NG, $\mathrm{MA}$, or $\mathrm{HG}$ for 12,24 , or $48 \mathrm{~h}(n=3)$. Bars represent mean \pm S.E.M. ${ }^{*} P<0.05,{ }^{\star \star} P<0.01,{ }^{* \star} P<0.001$ and ${ }^{\#} P<0.05$

(NG, normal glucose), $5.3 \mathrm{mM}$ glucose $+25 \mathrm{mM}$ mannitol (M, an osmotic control), or $30 \mathrm{mM}$ D-glucose ( $\mathrm{HG}$, high glucose) for $48 \mathrm{~h}$. Consistent with the TUNEL staining, flow cytometry analysis showed that the rate of apoptosis was significantly increased in HG-treated group compared that in control groups (Figure 1e). We then isolated the glomeruli from streptozotocin-induced diabetic rats for western blot analysis (Supplementary Figure 1). Result shown that the expression levels of Bax and cleaved caspase 3, two known indicators of apoptosis, were significantly increased in the obtained from streptozotocin-induced diabetic rats (Figures $1 \mathrm{f}-\mathrm{h}$ ), as well as in HG-induced MPC5 cells (Figures 1i-k), in comparison those in their respective controls.

\section{Mtdh expression is enhanced in vivo and in vitro models}

of DN. To determine whether Mtdh is expressed in podocytes, we performed double fluorescence staining of nephrin (podocyte marker) and Mtdh. Our results demonstrated that Mtdh is expressed not only in the tubules but also in the glomeruli of the investigated mice. Furthermore, the colocalization of Mtdh and nephrin indicated that Mtdh is predominantly expressed in glomerular podocytes. The intensity of Mtdh staining was shown to be much stronger in $\mathrm{db} / \mathrm{db}$ mice than in $\mathrm{db} / \mathrm{m}$ mice (Figure 2a). Western blot analyses demonstrated that Mtdh expression is significantly elevated in the DN glomeruli in comparison with that in the control
(Figure 2b). In addition, western blot analyses demonstrated that, following the treatment of MPC5 cells with $\mathrm{HG}$ (from 0 to $50 \mathrm{mM}$ range), the expression of Mtdh considerably increased (Supplementary Figure 2). Furthermore, Mtdh mRNA expression was significantly increased in HGinduced MPC5 cells in comparison with the control (Figure 2c). This was accompanied by a significant increase in the protein levels of Mtdh in HG-induced MPC5 cells at different time points $(12,24$, or $48 \mathrm{~h})$, although not in a time-dependent manner (Figure 2d).

Mtdh knockdown suppresses HG-induced MPC5 apoptosis, but Mtdh overexpression promotes apoptosis. We further investigated the role of Mtdh in the induction of apoptosis by Mtdh knockdown or overexpression. The results obtained using flow cytometry showed that the rate of apoptosis of $\mathrm{HG}$-induced MPC5 cells transfected with small interfering RNAs (siRNAs) targeting Mtdh (si-Mtdh) considerably decreased compared with the negative control (NC) group (Figure 3a). Successful Mtdh knockdown was confirmed by western blot analysis. Mtdh expression in HG-induced MPC5 cells was shown to be considerably decreased following the knockdown (Figures $3 b$ and c). In addition, the inhibition of Mtdh expression was shown to suppress the expression of Bax and cleaved caspase 3 in HG-induced MPC5 cells (Figures 3b, d, and e). 


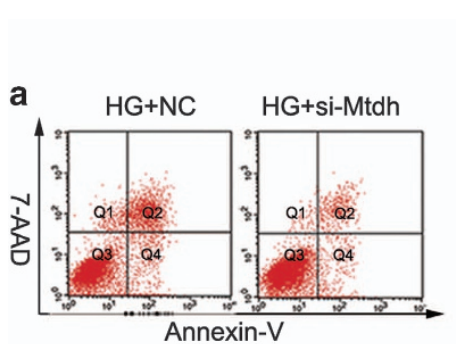

C

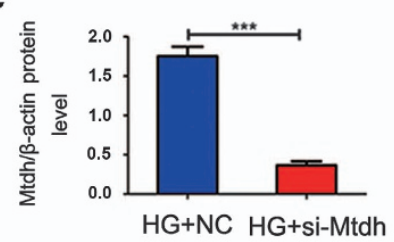

f

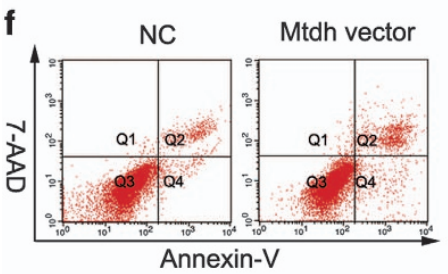

h

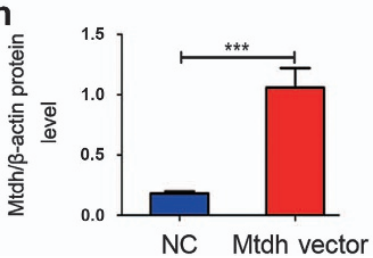

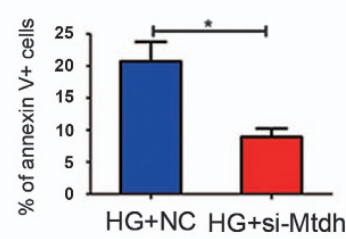

d

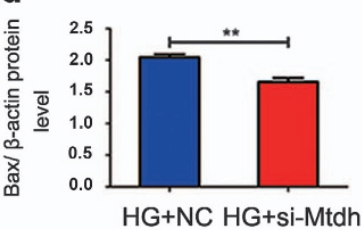

$\mathrm{HG}+\mathrm{NC} \mathrm{HG}+\mathrm{si}-\mathrm{Mtdh}$

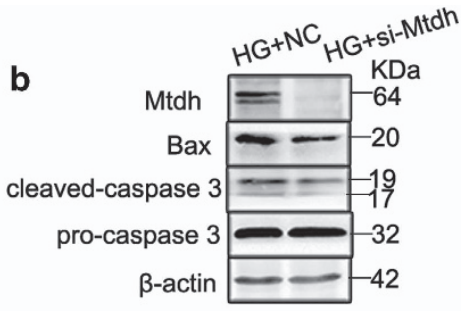

e
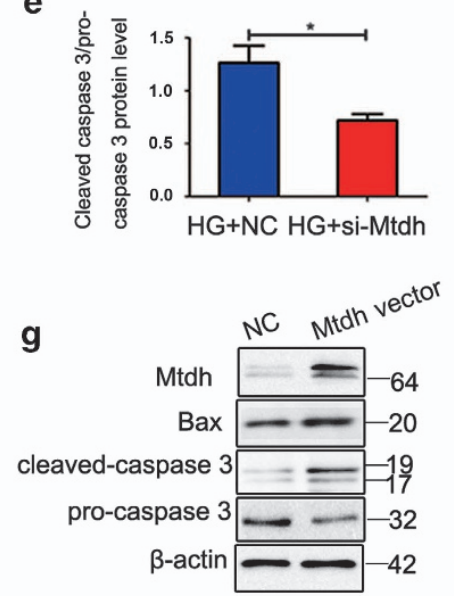

k

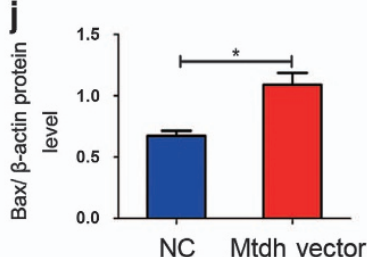

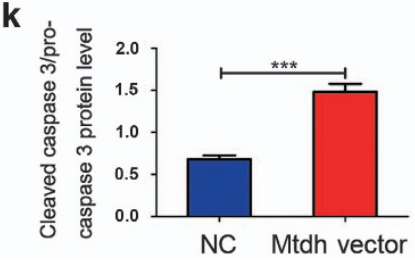

Figure 3 Mtdh expression levels affect the apoptosis of HG-induced MPC5 cells. (a) Flow cytometry analysis of the apoptosis rate of MPC5 cells transfected with NC or si-Mtdh and treated with HG for $48 \mathrm{~h}(n=3)$. (b) Mtdh, Bax, and cleaved caspase 3 protein expression levels in MPC5 cells following the transfection with NC or si-Mtdh and stimulation with HG for $48 \mathrm{~h}$. (c-e) Quantification of Mtdh (c), Bax (d), and cleaved caspase 3 (e) expression in MPC5 cells $(n=3)$. (f) Flow cytometry analysis of the apoptosis rate of MPC5 cells transfected with control vector (CV) or Mtdh overexpression vector $(n=3)$. (g) Mtdh, Bax, and cleaved caspase 3 protein expression levels in MPC5 cells following the transfection with CV or Mtdh overexpression vector. (h-k) Quantification of Mtdh (h), Bax (j), and cleaved caspase 3 (k) expression in MPC5 cells ( $n=3$ each). Bars represent mean \pm S.E.M. ${ }^{*} P<0.05,{ }^{\star \star} P<0.01$, and ${ }^{\star \star \star} P<0.001$

Following the overexpression of Mtdh in MPC5 cells, we showed that the apoptosis rate was significantly elevated in the Mtdh overexpression group compared with control vectortreated cells (Figure $3 \mathrm{f})$. Similarly, Mtdh overexpression led to an increase in both Bax and cleaved caspase 3 levels in MPC5 (Figures 3g-k).

Mtdh regulates MPC5 apoptosis through the p38 MAPK pathway. To investigate whether Mtdh-induced apoptosis of HG-induced MPC5 cells is mediated by the p38 MAPK pathway, we measured the expression of phosphorylated (p)-p38 MAPK in the glomeruli from DN rats and HG-induced MPC5 cells. The expression levels of p-p38 MAPK were shown to be significantly increased in the DN glomeruli (Figures $4 \mathrm{a}$ and $\mathrm{b}$ ) and HG-induced MPC5 cells (Figures 4c and d), in comparison with their respective controls. In addition, we showed that p38 MAPK pathway activation is involved in HG-induced MPC5 apoptosis, using p38
MAPK inhibitor, SB203580. Flow cytometry analysis showed that SB203580 treatment significantly inhibits HGinduced apoptosis in MPC5 cells (Figures $4 \mathrm{e}$ and $\mathrm{f}$ ). Furthermore, the suppression of p38 MAPK pathway activation by this inhibitor led to a decrease in Bax and cleaved caspase 3 expression levels in comparison with the control, although the basal levels of p-p38 did not change (Figures 4gj). MPC5 cells were then pretreated with si-Mtdh or NC, and treated with $\mathrm{HG}$ for $48 \mathrm{~h}$. As demonstrated, si-Mtdh treatment led to a decrease in p-p38 MAPK expression in comparison with the NC group (Figure 4k), and Mtdh overexpression was shown to increase the expression of p-p38 MAPK in comparison with the control group (Figure 4l).

Mtdh represents a direct target of the members of miR-30 family. The identification of putative Mtdh-regulating miRNAs, using TargetScan v5.1 (Cambridge, MA, USA), showed that $3^{\prime}$-untranslated region (UTR) of Mtdh contains 
a

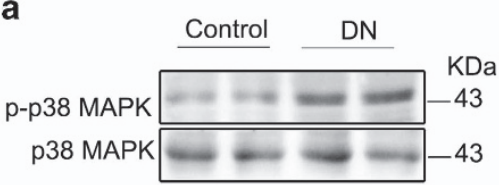

C

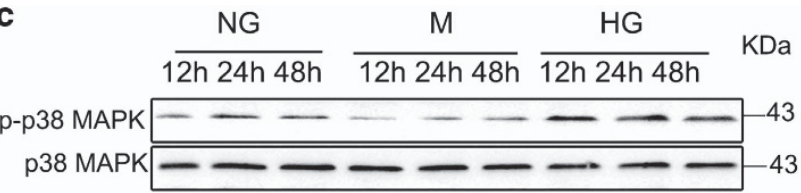

b

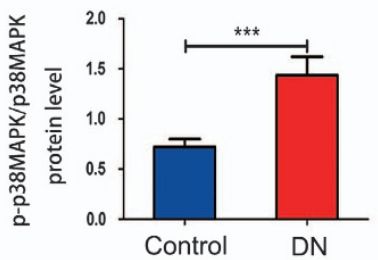

e

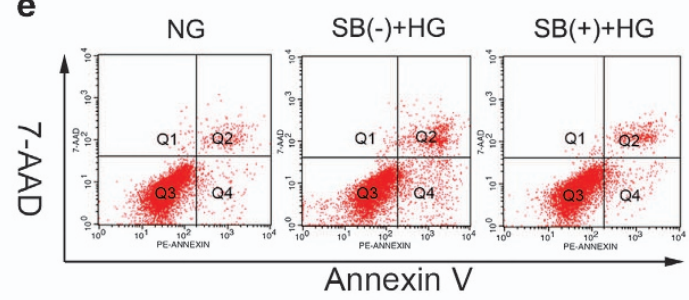

h

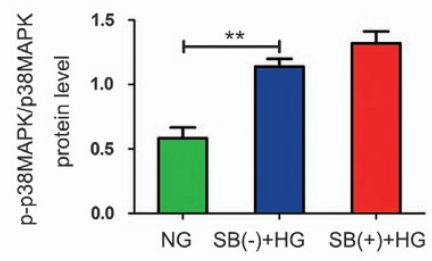

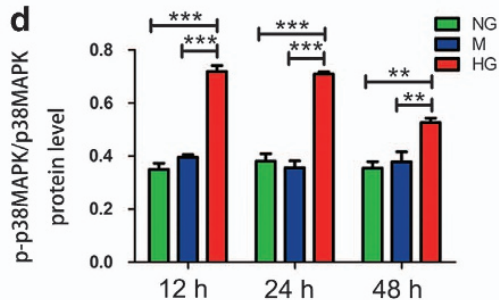

$12 \mathrm{~h} \quad 24 \mathrm{~h} \quad 48 \mathrm{~h}$
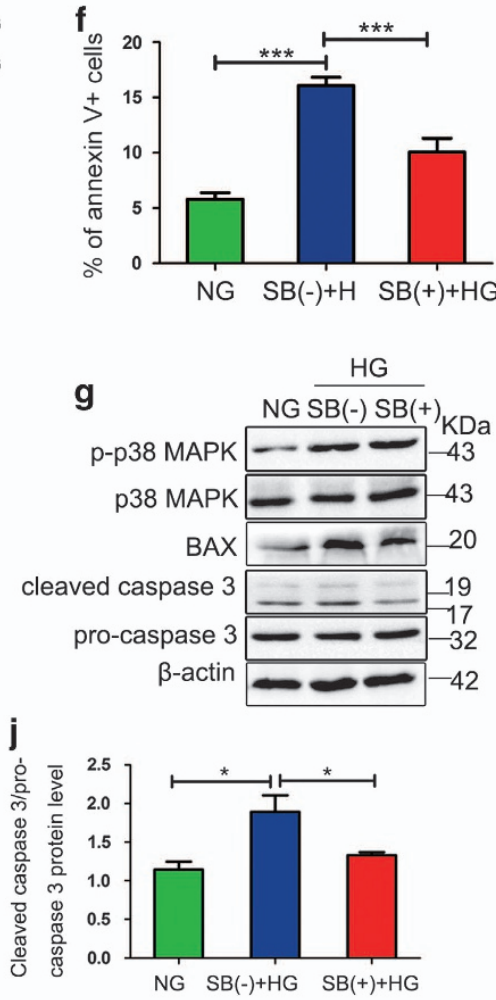

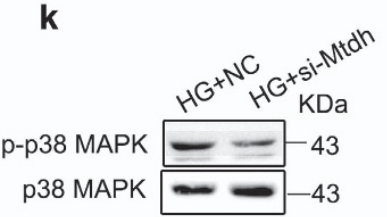

i

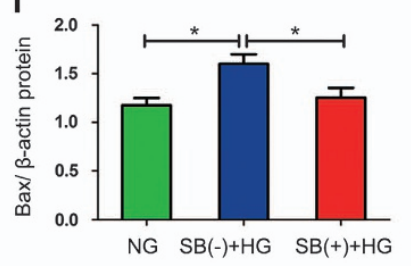

I

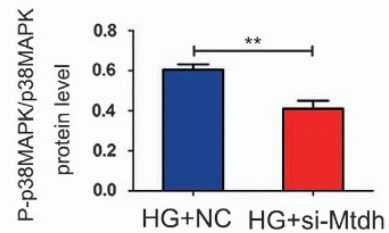

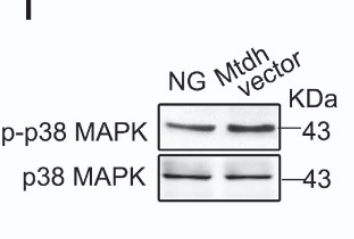

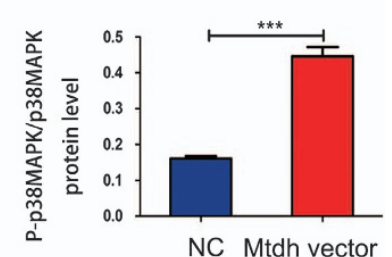

Figure 4 Mtdh facilitates MPC5 apoptosis through the activation of p38 MAPK pathway. (a) p-p38 MAPK expression in the samples obtained from the glomeruli of DN and control rat. (b) Quantification of the results presented in a $(n=6)$. (c) p-p38 MAPK expression in HG-induced MPC5 cells for 12, 24, and $48 \mathrm{~h}$. (d) Quantification of the results presented in $\mathbf{c}(n=3)$. (e) Apoptosis of MPC5 cells treated with NG, HG, and HG, and p38 MAPK inhibitor (p38l) for $48 \mathrm{~h}$. (f) Quantification of the results presented in $\mathbf{e}(n=3)$. (g) p-p38, Bax, and cleaved caspase 3 expression levels in MPC5 cells treated with NG, HG, and HG, and p38l for $48 \mathrm{~h}$. (h-j) Quantification of p-p38 MAPK (h), Bax (i), and cleaved caspase 3 (j) expressions ( $n=3)$. (k) p-p38 MAPK expression levels in NC or si-Mtdh-treated MPC5 incubated with HG for $48 \mathrm{~h}(n=3)$. (I) p-p38 MAPK expression levels in control vector or Mtdh overexpression vector-treated cells $(n=3)$. Bars represent mean \pm S.E.M. ${ }^{\star} P<0.05,{ }^{* \star} P<0.01$, and ${ }^{\star * \star} P<0.001$

seed sequences of miR-30s and that these sequences are highly conserved in diverse species (Figure 5a). Therefore, we studied miR-30 expression in the DN glomeruli and HGinduced MPC5 cells. As shown in Figure 5b, miR-30s were significantly downregulated in the DN glomeruli in comparison with the control. In addition, the levels of miR-30s were considerably decreased in MPC5 cells stimulated with HG for 12,24 , and $48 \mathrm{~h}$ (Figure $5 \mathrm{c}$ ). These results suggest that miR-30s may have a role in DN development.

Next, luciferase reporters, containing normal or mutated binding sites for miR-30s, were constructed. The obtained results demonstrated that $\mathrm{miR}-30 \mathrm{a},-30 \mathrm{~b},-30 \mathrm{c},-30 \mathrm{~d}$, and $-30 \mathrm{e}$ mimics can significantly inhibit the luciferase activity of the wild-type Mtdh $3^{\prime}$-UTR reporter, but not that of the NC, and that this inhibition was reduced when the mutant reporter, with mutated miR-30-binding site, was used (Figures $5 d-h$ ).

miR-30s lead to the reduction in Mtdh expression in HG-induced MPC5 cells. To assess the effects of miR-30s on the expression of Mtdh, we transiently transfected MPC5 cells with miR-30 inhibitors, synthetic miRNA mimics, or their NCs. As shown in Figures $6 a$ and $b$, transient 
a

GENE:

Mouse MTDH NM_178812 3'-UTR length:3444

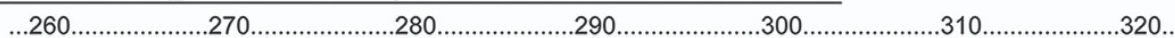

Mmu: UAUCCUGGUUUUACAACA--GUUACCUGUUUACAACAGACU--GUGCCCUGUCUCCUCUGCAGCUG

Hs a: UAUCCUGGUUUAACAACA--GUGCCCUGUUUACAACAGAUU--GUGCCCUAUCUCAUCUGCAGCCG

Rn o: UAUCCUGGUUUUACAACA--GUUCCCUGUUUACAACAGAUU--GUGCCCUGUUUCAUCUGCAGCUG

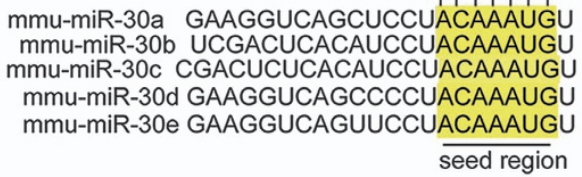

b

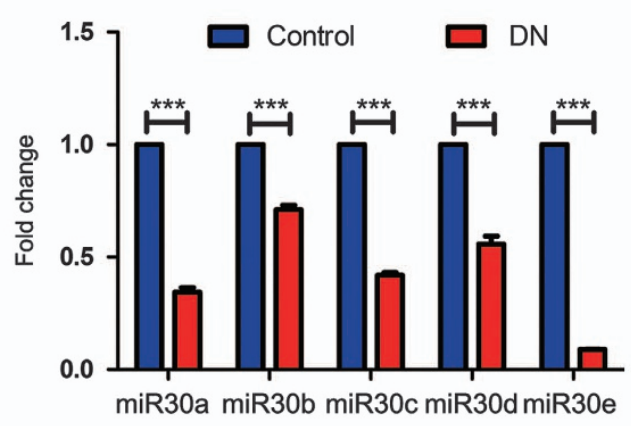

d

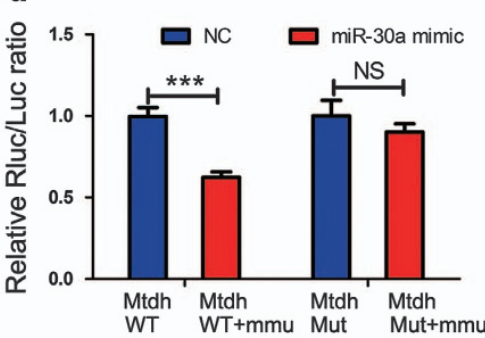

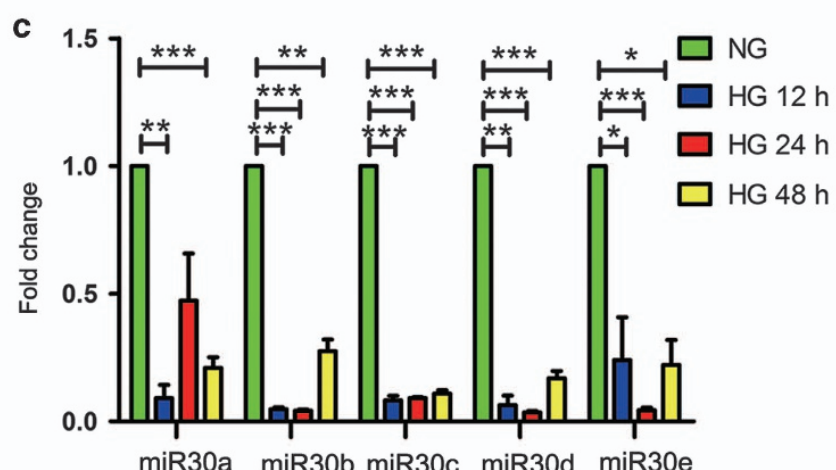

miR30a miR30b miR30c miR30d miR30e

e

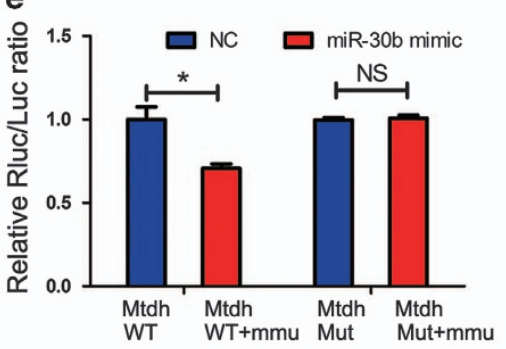

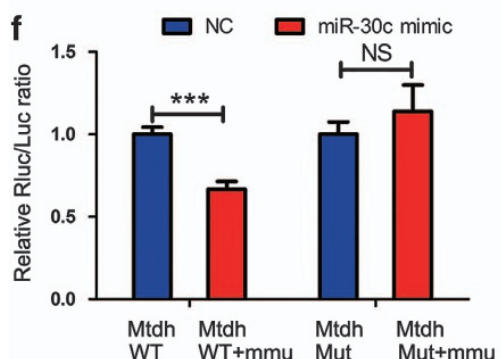
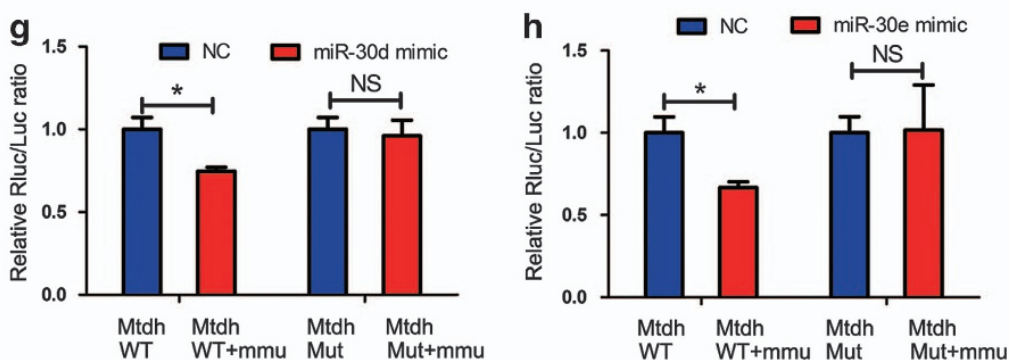

Figure 5 Mtdh represents a direct target of the members of miR-30 family. (a) Predicted target sequence of miR-30s in Mtdh 3'-UTR in different species and the mouse $3^{\prime}$-UTR was study in our experiment. (b and c) The expression of miR-30s in the glomeruli of DN or control (b) and HG-induced MPC5 cells in different time points $(0,12,24$, and $48 \mathrm{~h})$ (c). U6 was used for the normalization of the obtained levels $(n=3)$. (d-h) Luciferase activity measured in MPC5 co-transfected with miR-30a, $-30 \mathrm{~b},-30 \mathrm{c},-30 \mathrm{~d}$, and $-30 \mathrm{e}$ mimics, and NC, and the luciferase reporter for Mtdh wild-type (WT) or mutant (Mut) $3^{\prime}-$ UTR ( $n=3$ ). Bars represent mean \pm S.E.M. ${ }^{\star} P<0.05$, ${ }^{* \star} P<0.01$, and ${ }^{* \star *} P<0.001$

transfection of MPC5 cells with miR-30a inhibitor resulted in significant reduction of miR-30a expression, and miR-30a mimic treatment considerably increased miR-30a levels. Mtdh mRNA level was shown to be significantly increased following the treatment with miR-30 inhibitors (Figure 6c), whereas miR-30s mimics led to a considerable reduction of Mtdh expression induced by HG (Figure 6d), compared with the corresponding NC treatment groups. Mtdh protein expression was shown to be increased as well after the treatment with the miR-30 inhibitors (Figure 6e), whereas miR-30 mimics significantly reduced Mtdh expression in HG-induced MPC5 cells (Figure 6f).

miR-30s reduce the rate of HG-induced MPC5 apoptosis. MPC5 were transfected with miR-30 inhibitors, mimics, or the respective NCs. Flow cytometry analysis demonstrated that the transfection of cells with miR-30 inhibitors significantly 

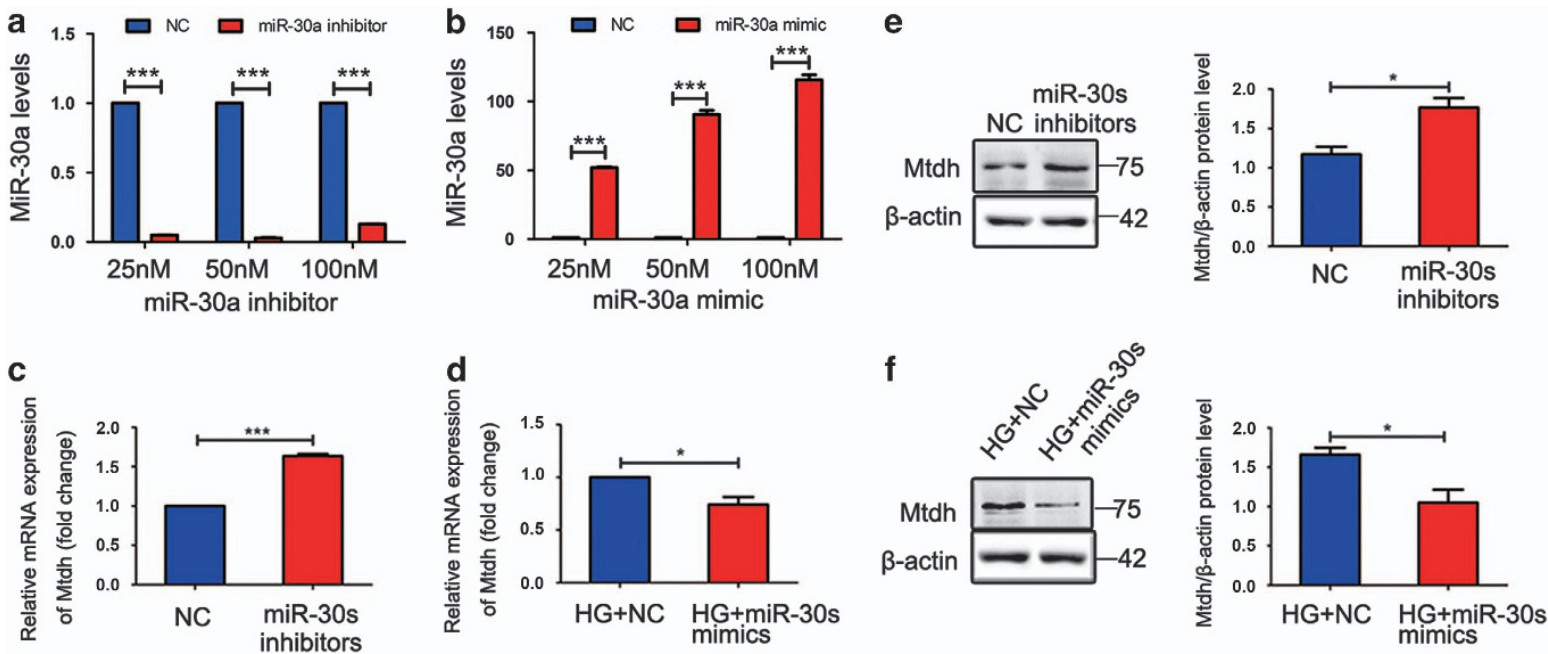

Figure 6 miR-30s inhibit Mtdh expression in HG-induced MPC5 cells. (a) miR-30a levels after the transient transfection of MPC5 cells with miR-30a inhibitor with different concentrations or their corresponding NCs $(n=3)$. (b) miR-30a levels after the transient transfection of MPC5 cells with miR-30a mimic with different concentrations or their corresponding NCs $(n=3)$. (c) Mtdh mRNA levels after the transient transfection of MPC5 cells with miR-30 inhibitors or their corresponding NCs $(n=3)$. (d) Mtdh mRNA levels in MPC5 cells after the transient transfection with miR-30 mimics or the corresponding NCs, and the following $48 \mathrm{~h}$ treatment with $\mathrm{HG}(n=4)$. (e) Mtdh protein expression levels in MPC5 cells treated as in $\mathbf{c}(n=3)$. (f) Mtdh protein expression levels in MPC5 cells treated as in $\mathbf{d}(n=3)$. Bars represent mean \pm S.E.M. ${ }^{*} P<0.05,{ }^{* \star} P<0.01$, and ${ }^{* \star *} P<0.001$

increased the percentage of apoptotic cells compared with the NC group (Figure 7a). Conversely, miR-30 mimics considerably reduced the rate of MPC5 apoptosis induced by $\mathrm{HG}$ (Figure 7b). Furthermore, miR-30 inhibitors significantly increased the expression of Bax and cleaved caspase 3 (Figure 7c). The treatment with miR-30 mimics considerably decreased HG-induced increase in the levels of these proteins (Figure 7d).

\section{Discussion}

Podocyte apoptosis is one of the major causes of podocyte loss and it has a crucial role in DN pathogenesis. ${ }^{1}$ Podocytes are exposed to various stimuli, such as hyperglycemia, fatty acids, growth factors, cytokines, and hormones, in diabetes mellitus. It has been demonstrated that, both in vivo and in vitro, HG levels lead to the activation of a series of event cascades in the kidneys, which may result in podocyte apoptosis. These may include increased oxidative stress, one of the most studied stimuli, ${ }^{29,30}$ the activation of RAS, the induction of TGF- $\beta$, and the formation of advanced glycation end products. ${ }^{31}$ Langer et al. ${ }^{32}$ and Huang et al. ${ }^{33}$ demonstrated that $\mathrm{HG}(30 \mathrm{mM})$ induces podocyte apoptosis in vitro. Consistent with this, we showed that the apoptosis rate of podocytes is significantly increased in $\mathrm{db} / \mathrm{db}$ mice, a wellrecognized mouse model of type 2 diabetes, and that the increase in glucose levels is sufficient to induce apoptosis in MPC5. However, we suggest a different mechanism of HGinduced podocyte apoptosis, supported by the results presented in this study that showed that the downregulation of miR-30s leads to Mtdh upregulation, inducing MPC5 apoptosis mediated by the activation of p38 MAPK pathway (Figure 8).

Previously, MTDH expression was determined in glucoseinduced proximal tubular epithelial cells. ${ }^{34}$ Recent studies have demonstrated that MTDH knockdown leads to the inhibition of cell growth and induces the apoptosis in human retinoblastoma as well as in hepatocellular carcinoma. ${ }^{35,36}$ Here we showed that Mtdh is expressed in the tubules as well as the glomeruli, predominantly in podocytes. Furthermore, Mtdh expression was shown to be increased in the renal tissues of $\mathrm{db} / \mathrm{db}$ mice, in the glomeruli form streptozotocininduced diabetic rats and in HG-induced MPC5, and that Mtdh inhibition leads to the reduction in HG-induced MPC5 apoptosis. These findings indicate that Mtdh has a crucial role as a positive regulator of HG-induced apoptosis in these cells.

Recently, numerous studies demonstrated that MTDH is involved in many signaling pathways including NF-KB, $\mathrm{PI}$ KK/AKT, Wnt/ $\beta$-catenin, MEK/ERK, and p38 MAPK pathways. ${ }^{15,16,37}$ In addition, the p38 MAPK pathway was shown to have an important role in podocyte injury. ${ }^{38,39}$ The data obtained in this study showed that the expression of p-p38 is significantly enhanced in the DN glomeruli and HGinduced MPC5 cells, and it is accompanied by the increase in the expression of Bax and cleaved caspase 3 . The inhibition of Mtdh led to the suppression of the activation of p38 MAPK pathway following the $\mathrm{HG}$ treatment, which suggests a possible link between Mtdh and p38 MAPK activation.

miRNAs were implicated in podocyte apoptosis as well. Eisenrech et al. ${ }^{17}$ demonstrated that miR-29b is involved in the regulation of apoptosis in podocytes through the regulation of podoplanin. Several miRNAs were found to regulate the expression of $\mathrm{MTDH}$, and they may be involved in the pathogenesis of different diseases. ${ }^{8}$ These miRNAs include miR-26a, miR-203, miR-22, miR-375, and other. ${ }^{16}$ miR-30s were demonstrated to have a protective role in podocyte injury and to be involved in actin fiber disruption and podocyte apoptosis. $^{28}$ Liu et al. ${ }^{40}$ suggested that $\mathrm{MTDH}$ can induce EMT in human non-small cell lung cancer through the regulation of miR-30a activity, whereas $\mathrm{He}$ et al. ${ }^{41}$ showed 

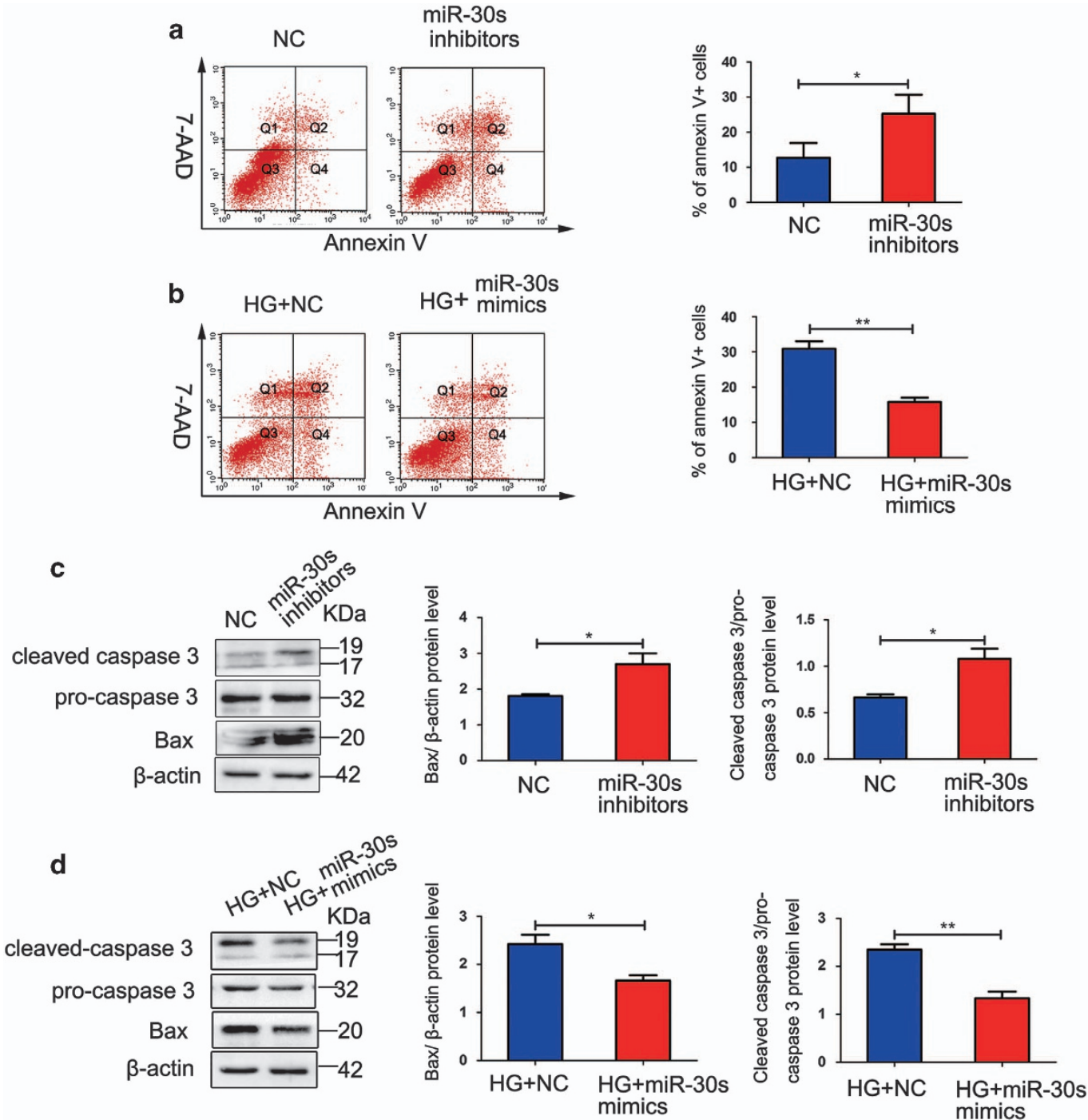

Figure 7 miR-30s reduce the rate of HG-induced MPC5 apoptosis. (a) miR-30 inhibitors significantly increase MPC5 apoptosis $(n=3)$. (b) miR-30 mimics inhibit HG-induced MPC5 apoptosis $(n=3)$. (c) miR-30 inhibitors induce the expression of Bax $(n=3)$ and cleaved caspase $3(n=4)$. (d) miR-30 mimics suppress the HG-induced upregulation of Bax and cleaved caspase $3(n=3)$. Bars represent mean \pm S.E.M. ${ }^{*} P<0.05,{ }^{* *} P<0.01$, and ${ }^{* \star *} P<0.001$

that miR-30a-5p suppresses the proliferation of hepatocellular carcinoma cells and enhances their apoptosis by targeting MTDH. A study by Li et al. ${ }^{27}$ showed that miR-30a-5p induced liver cancer cell apoptosis via the MTDH/PTEN/AKT pathway. We also found the activation of PTEN/AKT pathway in HGinduced MPC5 (Supplementary Figure 3). Here we confirmed that Mtdh represents a target of mi-R30s in DN, and that the expression of all five miR-30 family members is downregulated in the glomeruli form streptozotocin-induced diabetic rats and HG-induced MPC5 cells. In addition, our results agree with the results of a previous study, ${ }^{28}$ where miR-30s were found to protect podocytes from apoptosis induced by HG. However, the disagreement between the results of our study and the study conducted by He et al. ${ }^{41}$ and Li et al. ${ }^{27}$ may be due to the differences in the disease background and the alternative sources of the cells used in these studies.

In conclusion, we found that the upregulation of Mtdh facilitates podocyte apoptosis by inducing the activation of p38 MAPK signaling pathway. The obtained data indicate that miR-30s modulate Mtdh-induced podocyte apoptosis by targeting Mtdh 3'-UTR directly. Although the protective roles of miR-30s and Mtdh inhibition in DN have not been completely confirmed, we propose a novel cellular and molecular mechanism that may induce podocyte apoptosis in DN and provide new insights into the role of Mtdh.

\section{Materials and Methods}

Animal models. Diabetic db/db mice C57BL/KsJ (BKS.Cg-Dock $7^{\mathrm{m}}+/+$ Lepr ${ }^{\mathrm{db}} / \mathrm{J}$ ) and their normal littermates ( $\mathrm{db} / \mathrm{m} ; n=9$ in each group) of both sexes, aged 6 weeks, were purchased from the Model Animal Research Center at Nanjing University, China. During the experiments, all animals were housed in standard animal facilities, at $22-26{ }^{\circ} \mathrm{C}$ and $40-70 \%$ humidity, and a $12 / 12 \mathrm{~h} \mathrm{light/dark}$ cycle. All animal were killed at the age of 29 weeks. The study design and all animal procedures conformed to National Institutes of Health guidelines and were approved by the ethics committee for the experimental use of animals at Southern Medical University, Guangzhou, China (L2015046). 


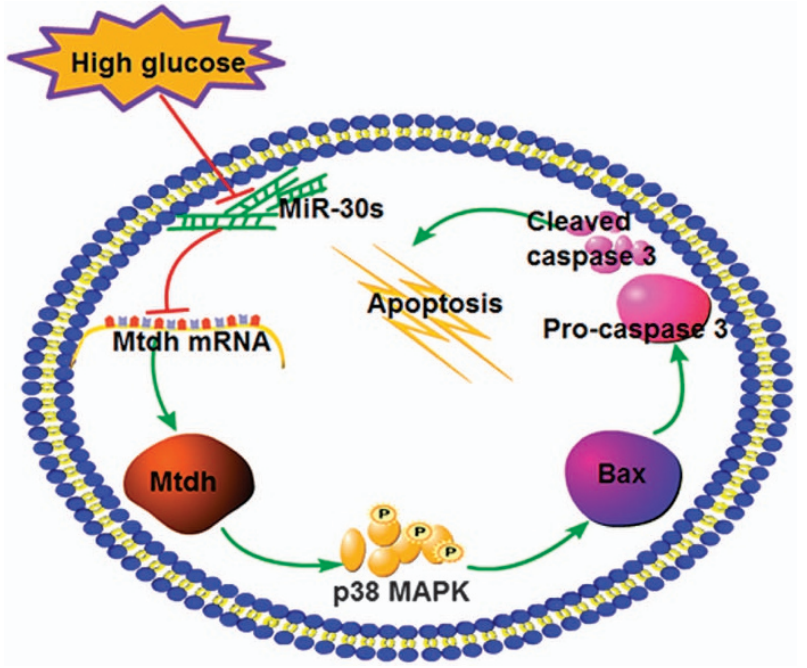

Figure 8 The proposed Mtdh-modulated apoptosis induction network in MPC5 cells miR-30s target Mtdh in HG-induced MPC5 cells. HG downregulates miR-30 family member expression and further increases Mtdh levels, which induces MPC5 apoptosis through the activation of p38 MAPK signaling pathway

Cell culture. Conditionally immortalized mouse podocytes (MPC5), between passages 12 and 20 (kindly provided by Professor Peter Mundel, Mount Sinai School of Medicine, USA, through Professor Wei Shi, Guangdong General Hospital, China), were cultured as previously described. ${ }^{42}$ In brief, undifferentiated podocytes were cultured in collagen I-coated dishes (BD Biosciences, Bedford, MA, USA) at $33^{\circ} \mathrm{C}$ (permissive temperature) and $5 \% \mathrm{CO}_{2}$ in RPMI 1640 (Gibco BRL, Gaithersburg, MD, USA) containing $10 \%$ fetal bovine serum (FBS; Gibco BRL), penicillin $(100 \mathrm{U} / \mathrm{ml})$, streptomycin (Gibco BRL; $100 \mathrm{mg} / \mathrm{ml}$ ), and $50 \mathrm{IU} / \mathrm{ml}$ of recombinant murine IFN- $\gamma$ (Peprotech, Rocky Hill, CT, USA). To induce differentiation, the cells were transferred to $37^{\circ} \mathrm{C}$ (nonpermissive temperature) for 10-14 days and the medium was replaced with RPMl 1640 containing 5\% FBS without IFN- $\gamma$. For the experiments, the medium was replaced by Dulbecco's modified Eagle medium (DMEM), containing $5.3 \mathrm{mM}$ glucose (Invitrogen, Carlsbad, CA, USA) supplemented with $1 \%$ FBS. The differentiated podocytes were treated with $5.3 \mathrm{mM}$ glucose (NG group), $5.3 \mathrm{mM}$ glucose $+25 \mathrm{mM}$ mannitol (M group; Beijing Dingguo Changsheng Biotechnology, Beijing, China), $30 \mathrm{mM}$ D-glucose (HG group; Sigma-Aldrich, St. Louis, MO, USA) for 12, 24, and $48 \mathrm{~h}$, or HG plus SB203580 (Abcam, Cambridge, USA; $10 \mu \mathrm{M}$ ) for $48 \mathrm{~h}$, depending on the experiment.

Apoptotic nucleus scoring in situ. To identify podocyte apoptosis in vivo, we performed double WT-1 and TUNEL ${ }^{7}$ staining. In brief, renal frozen sections were incubated with anti-WT-1 polyclonal antibody (1:50; Abcam) overnight at $4{ }^{\circ} \mathrm{C}$ After the washing, a fluorescent secondary antibody, Alexa Fluor 546 (1:1000; Invitrogen) was applied for $6 \mathrm{~h}$ at $4^{\circ} \mathrm{C}$, and the samples were rinsed with phosphate-buffered saline (PBS; Wuhan Boster Biological Technology, Wuhan, China). Afterward, TUNEL staining was performed using the In Situ Cell Death Detection kit (Roche Molecular Biochemicals, Mannheim, Germany), according to the manufacturer's protocol. The specimens were counterstained with 4',6-diamidino-2-phenylindole (DAPI; BestBio, Shanghai, China) for $10 \mathrm{~min}$, and following this, the images were obtained with a light microscope (Ni-U, Nikon Corporation, Tokyo, Japan). Podocyte apoptosis was defined as the double WT-1 and TUNEL staining of a cell, and double labeled/WT-1 staining ratio was calculated.

Flow cytometry. MPC5 apoptosis was analyzed by flow cytometry using the PE Annexin V Apoptosis Detection kit (BD Biosciences). MPC5 cells were incubated in six-well plates. Following the treatment with NG, M, or HG for $48 \mathrm{~h}$, or RNA transfection, the treated cells were collected, washed, and resuspended in $300 \mu \mathrm{l}$ of binding buffer, according to the manufacturer's instructions. The solution containing $3 \mu \mathrm{l}$ of PE Annexin and $3 \mu \mathrm{l}$ of 7-AAD was added, and the samples were incubated in the dark for $15 \mathrm{~min}$ at room temperature. These samples were analyzed using a cytometer (BD Biosciences).

Immunofluorescence analysis. Mtdh and nephrin (podocyte marker) antibodies were used to investigate the location and expression of Mtdh in frozen renal tissue samples obtained from $\mathrm{db} / \mathrm{db}$ and $\mathrm{db} / \mathrm{m}$ mice. The slides were permeabilized with $0.05 \%$ Triton X-100 (Biosharp, Anhui, China) in PBS for 10 min and blocked with $5 \%$ goat serum mixed with $2.5 \%$ bovine serum albumin for $2 \mathrm{~h}$. Afterward, these sections were incubated with anti-Mtdh antibody (1:100; Abcam) together with anti-nephrin antibody (1:50; Santa Cruz Biotechnology, Santa Cruz, CA, USA) at $4{ }^{\circ} \mathrm{C}$ overnight. Anti-Mtdh and anti-nephrin primary antibodies were detected using Alexa Fluor 546 donkey anti-rabbit and Alexa Fluor 488 goat anti-rabbit (1:1000; Invitrogen) secondary antibodies, respectively, and the samples were incubated with them for $6 \mathrm{~h}$ at $4{ }^{\circ} \mathrm{C}$. Cell nuclei were stained with DAPI for 10 min before the observation of the samples under a light microscope (Nikon Corporation).

Transient transfections with siRNAs, Mdth overexpression vector, miR-30 inhibitors, and miR-30 mimics. MPC5 were seeded at $2 \times 10^{5}$ cells per well in six-well plates at $70 \%$ confluence after the differentiation. These cells were transfected with Mtdh siRNA ( $50 \mathrm{nM}$; GenePharma, Shanghai, China) or the overexpression ( $500 \mathrm{ng}$ per well, GenePharma) the mixture of the mimics or inhibitors (RiboBio, Guangzhou, China) of all five miR-30 family members at the final concentrations of $50 \mathrm{nM}$, using Lipofectamine 2000 (Invitrogen) for $6 \mathrm{~h}$ in OPTI-MEM (Gibco BRL), according to the manufacturers' instructions. Afterward, OPTI-MEM was replaced with the complete medium containing $1 \% \mathrm{FBS}$, and treated with $\mathrm{HG}$ for $48 \mathrm{~h}$ after the transfection with siRNAs or mimics, whereas the cells treated with miR-30 inhibitors were not treated with $\mathrm{HG}$. The sequences of Mtdh siRNAs were as follows: Mtdh 1: sense: $5^{\prime}$-GGAUGAAGUUGUUAGAAAUTT -3', antisense: 5'-AUUUCUAACAACUUCAUCCTTUTT-3'; Mtdh 2: sense: 5'GUCUACCACUUCUGAUUAUTTUTT-3', antisense: 5'-AUAAUCAGAAGUGGUA GACTT-3'; Mtdh 3: sense: 5'-CGAGCCAUCUAUUACCUUATT-3', antisense: 5'UAAGGAAUAGAUGGCUCGTT-3'; and control: sense: $5^{\prime}$-UUCUCCGAA CGUGUCACGUTT-3', antisense: 5'-ACGUGACACGUUCGGAGAATT-3'.

RNA extraction and qPCR. miRNAs and total RNA were extracted using TRIzol reagent (TransGen Biotech, Beijing, China), and qPCR was performed using a commercial kit (Takara Biotechnology, Dalian, China) according to the manufacturer's protocol. All experiments were performed in triplicate and the comparative assessment of target gene expression was performed using $\beta$-actin mRNA or U6 miRNA levels as internal controls. The primers used in this study are listed in Supplementary Information 1. Changes in levels analyzed by the relative quantification $(\Delta \Delta \mathrm{Ct})$ method.

Isolation of glomeruli. Glomeruli were isolated from streptozotocin-induced diabetic rats (approved by the ethics committee for the experimental use of animals at Southern Medical University, Guangzhou, China (L2015007) and kindly provided by Professor Hong-xin Niu form ZhuJiang Hospital, Southern Medical University, China) using the standard mechanical sieving technique, as previously described. ${ }^{43}$ In brief , after mincing and grinding of the tissue, the renal cortex paste was forced through a series of mesh filters with 180-, 100-, 75-, and 70- $\mu$ m mesh openings. The tissue material remaining on the last sieve was rinsed and collected.

Western blotting. Protein samples were prepared using radioimmunoprecipitation assay buffer, and protein concentrations were determined using the BCA kit (Thermo Fisher Scientific, Waltham, MA, USA). Equal amounts of proteins $(50 \mu \mathrm{g})$ were separated on the $12 \%$ sodium dodecyl sulfate-polyacrylamide gels, and transferred to polyvinylidene fluoride membranes (Millipore, Bedford, MA, USA). Following the blocking with $5 \%$ skimmed milkTBST for $1 \mathrm{~h}$, the membranes were incubated overnight at $4{ }^{\circ} \mathrm{C}$ with primary antibodies against Mtdh (1:800; Abcam), p38 MAPK, phosphorylated (p)-p38 MAPK, pro-caspase 3, and cleaved caspase 3 (1:1000; Cell Signaling Technologies, Danvers, MA, USA), $\beta$-actin (1:1000; EarthOx LLC, San Francisco, CA, USA), and Bax (1:800; Cell Signaling Technologies). The membranes were probed with the appropriate HRP-conjugated secondary antibodies (1:10 000; EarthOx) for $1 \mathrm{~h}$ at room temperature. Protein band intensities were quantified as described previously.

Dual-luciferase reporter assay. The $3^{\prime}-U T R$ of Mtdh containing putative miR-30-binding sites was amplified and cloned into PmiR-RB-REPORT 
dual-luciferase reporter vector (RiboBio). In addition, a mutated Mtdh 3'-UTR was constructed (TGTTTAC (26-33, 284-291, 1547-1554) to ACAATT; GTTTAC (563-569) to CAAATG). MPC5 cells in logarithmic growth phase were seeded into 96-well plates and incubated for $48 \mathrm{~h}$ before the transfection. miRNA mimics or NCs and luciferase reporter vector containing the $3^{\prime}$-UTR of Mtdh or the mutant sequence vector were co-transfected into the cells using Lipofectamine 2000 transfection reagent (Invitrogen) in OPTI-MEM (Gibco BRL). Cells were collected $48 \mathrm{~h}$ after the transfection and dual-luciferase reporter assay was performed (Promega, Madison, WI, USA). Firefly luciferase activity was normalized to the corresponding levels of renilla luciferase activity.

Statistical analysis. Statistical analyses were performed with SPSS for Windows version 19.0 (SPSS, Chicago, IL, USA). All data were from at least three independent experiments. Data are presented as mean \pm S.E.M. Student's $t$-test was used for analyzing the differences between two groups, whereas ANOVA was used for the comparisons between multiple groups, followed by Student's $t$-test for the determination of differences between groups. $P<0.05$ was considered statistically significant in all cases.

\section{Conflict of Interest}

The authors declare no conflict of interest.

Acknowledgements. This work was supported by the National Natural Science Foundation of China (grant numbers 81072848 and 81673792 to Hai-Bo Long), the Science and Technology Planning Project of Guangdong Province, China (grant numbers 2013B021800149, 2014A020210011, and 2015A020211012 to Hai-Bo Long), the Natural Science Foundation of Guangdong Province, China (grant number 2014A030310065 to Fen-Fen Peng), and the Science and Technology Planning Project of Guangzhou, China (grant number 201510010137 to Hai-Bo Long). We thank Professor Wei Shi for providing the MPC5 cell line and professor Hong-xin Niu form ZhuJiang Hospital, Southern Medical University, China, for providing us with the renal tissue form streptozotocin-induced diabetic rats.

1. Shankland SJ. The podocyte's response to injury: role in proteinuria and glomerulosclerosis. Kidney Int 2006; 69: 2131-2147.

2. Susztak K, Raff AC, Schiffer M, Bottinger EP. Glucose-induced reactive oxygen species cause apoptosis of podocytes and podocyte depletion at the onset of diabetic nephropathy. Diabetes 2006; 55: 225-233

3. Tanaka E, Asanuma K, Kim E, Sasaki Y, Oliva Trejo JA, Seki T et al. Notch2 activation ameliorates nephrosis. Nat Commun 2014; 5: 3296.

4. Li G, Li Y, Liu S, Shi Y, Chi Y, Liu G et al. Gremlin aggravates hyperglycemia-induced podocyte injury by a TGFbeta/smad dependent signaling pathway. J Cell Biochem 2013. 114: 2101-2113

5. Schiffer M, Bitzer M, Roberts ISD, Kopp JB, ten Dijke P, Mundel P et al. Apoptosis in podocytes induced by TGF- $\beta$ and Smad7. J Clin Invest 2001; 108: 807-816.

6. Brown DM, Ruoslahti E. Metadherin, a cell surface protein in breast tumors that mediates lung metastasis. Cancer Cell 2004; 5: 365-374.

7. Britt DE, Yang DF, Yang DQ, Flanagan D, Callanan H, Lim YP et al. Identification of a novel protein, LYRIC, localized to tight junctions of polarized epithelial cells. Exp Cell Res 2004; 300: 134-148.

8. Sarkar D, Fisher PB. AEG-1/MTDH/LYRIC: clinical significance. Adv Cancer Res 2013; 120: 39-74.

9. Sarkar D, Emdad L, Lee SG, Yoo BK, Su ZZ, Fisher PB. Astrocyte elevated gene-1: far more than just a gene regulated in astrocytes. Cancer Res 2009; 69: 8529-8535.

10. Jeon HY, Choi M, Howlett EL, Vozhilla N, Yoo BK, Lloyd JA et al. Expression patterns of astrocyte elevated gene-1 (AEG-1) during development of the mouse embryo. Gene Expr Patterns 2010; 10: 361-367.

11. Lee SG, Kim K, Kegelman TP, Dash R, Das SK, Choi JK et al. Oncogene AEG-1 promotes glioma-induced neurodegeneration by increasing glutamate excitotoxicity. Cancer Res 2011: 71: 6514-6523.

12. Khuda II, Koide N, Noman AS, Dagvadorj J, Tumurkhuu G, Naiki Y et al. Astrocyte elevated gene-1 (AEG-1) is induced by lipopolysaccharide as toll-like receptor 4 (TLR4) ligand and regulates TLR4 signalling. Immunology 2009; 128: e700-e706.

13. Vartak-Sharma N, Ghorpade A. Astrocyte elevated gene-1 regulates astrocyte responses to neural injury: implications for reactive astrogliosis and neurodegeneration. $J$ Neuroinflammation 2012; 9: 195.
14. Vartak-Sharma N, Gelman BB, Joshi C, Borgamann K, Ghorpade A. Astrocyte elevated gene- 1 is a novel modulator of HIV-1-associated neuroinflammation via regulation of nuclear factor-kappaB signaling and excitatory amino acid transporter-2 repression. J Biol Chem 2014; 289: 19599-19612

15. Wei J, Li Z, Chen W, Ma C, Zhan F, Wu W et al. AEG-1 participates in TGF-beta1-induced EMT through p38 MAPK activation. Cell Biol Int 2013; 37: 1016-1021.

16. Lee SG, Kang DC, DeSalle R, Sarkar D, Fisher PB. AEG-1/MTDH/LYRIC, the beginning: initial cloning, structure, expression profile, and regulation of expression. Adv Cancer Res 2013; 120: 1-38.

17. Eisenreich $A$, Langer $S$, Herlan $L$, Kreutz R. Regulation of podoplanin expression by microRNA-29b associates with its antiapoptotic effect in angiotensin II-induced injury of human podocytes. J Hypertens 2016; 34: 323-331.

18. Kim EK, Choi EJ. Pathological roles of MAPK signaling pathways in human diseases. Biochim Biophys Acta 2010; 1802: 396-405.

19. Cuadrado A, Nebreda AR. Mechanisms and functions of p38 MAPK signalling. Biochem $J$ 2010; 429: 403-417.

20. Park IJ, Kim MJ, Park OJ, Park MG, Choe W, Kang I et al. Cryptotanshinone sensitizes DU145 prostate cancer cells to Fas(APO1/CD95)-mediated apoptosis through Bcl-2 and MAPK regulation. Cancer Lett 2010; 298: 88-98.

21. Gao K, Chi Y, Sun W, Takeda M, Yao J. 5'-AMP-activated protein kinase attenuates adriamycin-induced oxidative podocyte injury through thioredoxin-mediated suppression of the apoptosis signal-regulating kinase 1-P38 signaling pathway. Mol Pharmacol 2014; 85: $460-471$.

22. Saurus $\mathrm{P}$, Kuusela $\mathrm{S}$, Lehtonen $\mathrm{E}$, Hyvonen ME, Ristola M, Fogarty $\mathrm{CL}$ et al. Podocyte apoptosis is prevented by blocking the Toll-like receptor pathway. Cell Death Dis 2015; 6 : e1752.

23. Hwang HW, Mendell JT. MicroRNAs in cell proliferation, cell death, and tumorigenesis. Br J Cancer 2007; 96 Suppl R40-R44.

24. Zhang J, Zhang H, Liu J, Tu X, Zang Y, Zhu J et al. miR-30 inhibits TGF-beta1-induced epithelial-to-mesenchymal transition in hepatocyte by targeting Snail1. Biochem Biophys Res Commun 2012; 417: 1100-1105.

25. Zou Z, Wu L, Ding H, Wang Y, Zhang Y, Chen X et al. MicroRNA-30a sensitizes tumor cells to cis-platinum via suppressing beclin 1-mediated autophagy. J Biol Chem 2012; 287: $4148-4156$.

26. Li J, Donath S, Li Y, Qin D, Prabhakar BS, Li P. miR-30 regulates mitochondrial fission through targeting p53 and the dynamin-related protein-1 pathway. PLoS Genet 2010; 6: e1000795.

27. Li WF, Dai H, Ou Q, Zuo GQ, Liu CA. Overexpression of microRNA-30a-5p inhibits liver cancer cell proliferation and induces apoptosis by targeting MTDH/PTEN/AKT pathway. Tumour Biol 2016; 37: 5885-5895

28. Wu J, Zheng C, Fan Y, Zeng C, Chen Z, Qin W et al. Downregulation of microRNA-30 facilitates podocyte injury and is prevented by glucocorticoids. J Am Soc Nephrol 2014; 25: 92-104.

29. Lee SC, Han SH, Li JJ, Lee SH, Jung DS, Kwak SJ et al. Induction of heme oxygenase-1 protects against podocyte apoptosis under diabetic conditions. Kidney Int 2009; 76 : 838-848.

30. Eid AA, Gorin Y, Fagg BM, Maalouf R, Barnes JL, Block $\mathrm{K}$ et al. Mechanisms of podocyte injury in diabetes: role of cytochrome P450 and NADPH oxidases. Diabetes 2009; 58 : 1201-1211.

31. Anil Kumar P, Welsh Gl, Saleem MA, Menon RK. Molecular and cellular events mediating glomerular podocyte dysfunction and depletion in diabetes mellitus. Front Endocrinol (Lausanne) 2014; 5: 151.

32. Langer S, Kreutz R, Eisenreich A. Metformin modulates apoptosis and cell signaling of human podocytes under high glucose conditions. J Nephrol 2016; 29: 765-773.

33. Huang Z, Zhang L, Chen $Y$, Zhang $H$, Zhang $Q$, Li R et al. Cdc42 deficiency induces podocyte apoptosis by inhibiting the Nwasp/stress fibers/YAP pathway. Cell Death Dis 2016; 7: e2142.

34. Li WN, Wei JL, Wu M, Wu W, Huang Y, Xie MW et al. AEG-1 participates in high glucoseinduced activation of Rho kinase and epithelial-mesenchymal transition in proximal tubular epithelial cells. Asian Pac J Trop Med 2015; 8: 1076-1078.

35. Chang Y, Li B, Xu X, Shen L, Bai H, Gao F et al. Lentivirus-mediated knockdown of astrocyte elevated gene-1 inhibits growth and induces apoptosis through MAPK pathways in human retinoblastoma cells. PLOS One 2016; 11: e0148763.

36. Li WF, Ou Q, Dai H, Liu CA. Lentiviral-mediated short hairpin RNA knockdown of MTDH inhibits cell growth and induces apoptosis by regulating the PTEN/AKT pathway in hepatocellular carcinoma. Int J Mol Sci 2015; 16: 19419-19432.

37. Emdad L, Das SK, Dasgupta S, Hu B, Sarkar D, Fisher PB. AEG-1/MTDH/LYRIC: signaling pathways, downstream genes, interacting proteins, and regulation of tumor angiogenesis. Adv Cancer Res 2013; 120: 75-111.

38. Tian J, Zhang L, Zhou Y, Xiao J, Li S, Chen Y et al. Angiotensin-(1-7) attenuates damage to podocytes induced by preeclamptic serum through MAPK pathways. Int J Mol Med 2014; 34: 1057-1064.

39. Chen Z, Wan X, Hou Q, Shi S, Wang L, Chen P et al. GADD45B mediates podocyte injury in zebrafish by activating the ROS-GADD45B-p38 pathway. Cell Death Dis 2016; 7: e2068. 
40. Liu K, Guo L, Guo Y, Zhou B, Li T, Yang H et al. AEG-1 3'-untranslated region functions as a ceRNA in inducing epithelial-mesenchymal transition of humannon-small cell lung cancer by regulating miR-30a activity. Eur J Cell Biol 2015; 94: 22-31.

41. He R, Yang L, Lin X, Chen X, Lin X, Wei F et al. MiR-30a-5p suppresses cell growth and enhances apoptosis of hepatocellular carcinoma cells via targeting AEG-1. Int J Clin Exp Pathol 2015; 8: 15632-15641.

42. Shankland SJ, Pippin JW, Reiser J, Mundel P. Podocytes in culture: past, present, and future. Kidney Int 2007; 72: 26-36.

43. Fong JS, Drummond KN. Method for preparation of glomeruli for metabolic studies. J Lab Clin Med 1968; 71: 1034-1039.

44. Chen XW, Liu WT, Wang YX, Chen WJ, Li HY, Chen YH et al. Cyclopropanyldehydrocostunolide LJ attenuates high glucose-induced podocyte injury by suppressing RANKL/ RANK-mediated NF-kappaB and MAPK signaling pathways. J Diabetes Complications 2016; 30: 760-769. (c) (i) Cell Death and Disease is an open-access journal published by Nature Publishing Group. This work is licensed under a Creative Commons Attribution 4.0 International License. The images or other third party material in this article are included in the article's Creative Commons license, unless indicated otherwise in the credit line; if the material is not included under the Creative Commons license, users will need to obtain permission from the license holder to reproduce the material. To view a copy of this license, visit http://creativecommons.org/licenses/by/4.0/

(C) The Author(s) 2016

Supplementary Information accompanies this paper on Cell Death and Disease website (http://www.nature.com/cddis) 